\title{
Points in topoi of sheaves over distributive lattices
}

\author{
M. Adelman
}

This article generalizes the well known theorem that the geometric morphisms from the category of sets to a category of set-valued sheaves on a topological space correspond to the irreducible components of the topological space. As irreducible components are not available in any topos more general than a spatial one, they are characterized in terms of filters of open sets - which are available in any topos. It is then seen that the theorem phrased in these terms generalizes to sheaves on any lattice with reasonable distributivity conditions.

\section{0 . Introduction}

This paper is motivated by a representation of the traditional stalk functor from a spatial topos to the category of sets. Let $X$ be a topological space and let $x \in X$. Let $N_{x}$ denote the set of open neighbourhoods of $x$. Let $O X$ denote the poset of open subsets of $X$. Then the existence of intersections and a maximum element of $O X$ guarantees that $O X$ has finite limits. Define a functor $F: O \dot{X} \rightarrow$ Set (the category of sets) by

$$
F U= \begin{cases}1 \text { if } U \in N_{x} \\ 0 \text { otherwise. }\end{cases}
$$

Let $Y: O X \rightarrow \operatorname{Sh} v(X)$ be the inclusion of $U$ as the sheaf:

Received 14 March 1979. 


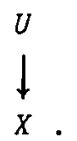

Then the stalk functor at $x$ is simply the left Kan extension of $F$ along $Y$. A well-known theorem guarantees this to be left exact since $F$ is. The stalk functor is known to have a right adjoint and hence establishes a point of $\operatorname{Shv}(X)$.

The above process can be repeated for any filter on $X$. This will give a left-exact functor from $\operatorname{Shv}(X)$ to Set. The problem is to determine which filters give rise to left exact functors with a right adjoint. If this is to work, in a class of topoi broader than spatial. topoi, the description of these filters can not make reference to an element $x$. The correct class of filters are those called constrained: filters. The general theorem is that the points in a category of set valued sheaves over a lattice with sufficient distributivity properties correspond to the constrained filters on this lattice.

If one wants to generalize further to categories of sheaves over a site whose underlying category is not a lattice, then one must dispense with filters. The filters arise because the category of elements of a left exact functor from a lattice to the category of sets is nothing but a filter on the lattice. Further generalizations would require replacing filters by cofiltered discrete fibrations.

The general method used here would work for any Grothendieck topology in which representable functors are sheaves. The problem is: given a flat functor $F: C \rightarrow$ Set and a Grothendieck topology $T$ on $C$, what are the conditions on $F$ or the category of elements of $F$ so that $\operatorname{Set}(F-, A)$ is a sheaf for all sets $A$ ?

\section{Points in categories of sheaves of distributive lattices}

THEOREM 1.1. Let $E$ be an elementary topos. Let $x$ denote the poset of subobjects of 1 (the terminal object of $E$ ). Then the points of $\left[\mathrm{X}^{\mathrm{Op}}\right.$, Set $]$ are the filters on $x$.

Proof. Filters to points. Let $F$ be a filter. Define $F: X \rightarrow$ Set by 


$$
F(U)=\left\{\begin{array}{l}
1 \text { if } U \in X, \\
0 \text { if } U \leqslant x .
\end{array}\right.
$$

Let $Y$ denote the Yoneda functor $X \rightarrow\left[X^{\mathrm{pp}}\right.$, Set $]$ and let $F^{*}$ be the left Kan extension of $F$ along $Y$ ([2], p. 232). Because $F$ is left exact, $F^{*}$ is left exact $([3]$, p. 200), and it always has a right adjoint $F_{*}:$ Set $\rightarrow\left[X^{\mathrm{p}}\right.$, Set $]$. Hence $F_{*}$ is a point of $\left[\mathrm{X}^{\mathrm{OP}}\right.$, Set $]$.

Points to filters. Let $P^{*} \rightarrow P_{*}$ be a point of $\left[\mathrm{X}^{\mathrm{OP}}\right.$, Set] . Let $F: X \rightarrow$ Set be $F=P^{*} Y$. Then $F$ is left exact and hence

$$
F=\{U \in X \mid F U=1\}
$$

is a filter on $X$. The density ([2], p. 242) of the Yoneda functor gives that these two processes are inverse of each other.

In the light of this theorem, one may attempt to phrase the problem of finding points of a topos $E$ as: which points (filters) of $\left[x^{\circ p}\right.$, Set] are points of $E$ ? The question at this level is meaningless as $E$ does not "lie in" $\left[\mathrm{X}^{\mathrm{op}}\right.$, Set $]$.

DEFINITION 1.2. A strongly distributive lattice is a lattice with the additional property that given $\left\{U_{\alpha}\right\}$ is a family in $L$ and $V \in L$ then $U_{\alpha} V \wedge U_{\alpha}$ exists if $\bigcup_{\alpha}^{U} U_{\alpha}$ exists and $V \wedge \underset{\alpha}{U} U_{\alpha}=\underset{\alpha}{U}\left(U_{\alpha} \wedge U\right)$. The cannonical Grothendieck topology on $L$ is given by specifying that the coverings of $U$ are families $\left\{U_{\alpha}\right\}$ so that $U=\underset{\alpha}{U} U_{\alpha}$.

Any Heyting algebra is a strongly distributive lattice.

DEFINITION 1.3. Let $P$ be a poset and $F$ a filter on $P$. Then $F$ is said to be constrained if the complement of $F$ in $P$ is closed under all unions. That is if $\left\{U_{\alpha}\right\} \subset P$ and $U_{\alpha} \notin F$ for all $\alpha$ then $U U_{\alpha} \notin F$. This includes the empty union so it implies $0 \notin F$ where 0 denotes the minimum element of $P$.

EXAMPLES 1.4. Let $N$ be a countable set. Let $L=P(N)$ (the lattice of subsets of $N$ ). This is a strongly distributive lattice. Let 
$F$ be the Fréchet filter (complements of finite sets). Then $F$ is not a constrained filter. For

$$
U\{U \mid U \notin F\}=U\{U \mid \text { complement of } U \text { is infinite }\}=N \in F .
$$

One can prove that for any set $X$, the constrained filters on $P X$ are the principal ultra-filters on $X$. More generally:

PROPOSITION 1.5. Let $X$ be a topological space and let

$$
L=O X=\text { the lattice of open subsets of } X \text {. }
$$

Then the constrained filters on $L$ are exactly those of the form

$$
F=\{U \mid U \notin W\}
$$

where $K$ is an irreducible subset of $X([1], 7.21)$ and $W=X-K$.

Proof. Let $W, K$, and $F$ be as in the hypothesis. It must first be shown that $F$ is a filter. The difficult property is the intersection property. Suppose $U \notin W$ and $V \notin W$ but $U \cap V \subset W$. Then

$$
K=X-W \subset(X-U) \cup(X-V) \text {; }
$$

hence

$$
K=[(X-U) \cap K] \cup[(X-V) \cap K] .
$$

Since $K$ is irreducible, one of the terms of the union is $K$, say

$$
K=(X-U) \cap K \text {. }
$$

Then $K \subset X-U$ or $U \subset W$ which is a contradiction. Hence $F$ is a filter.

To show that $F$ is constrained, note that $W=U\{U \mid U \subset W\}$; as this is contained in $W$ it is not in $F$. Let $\left\{U_{\alpha}\right\}$ be a family of open sets not in $F$. Then $U_{\alpha} \subset W$ for all $\alpha$. Hence $U_{\alpha} U_{\alpha} \subset W$ if $U_{\alpha} U_{\alpha}$ were in $F$ so would be $W$, which is a contradiction.

Conversely, given $F$ a constrained filter, let $W=U\{U \mid U \notin F\}$; then $W \notin F$. Let $K=X-W$; then $K$ is irreducible, for if $K=C \cup D$, then

$$
W=(X-C) \cap(X-D) \text {. }
$$

If both $X-C$ and $X-D$ were in $F$, then $W$ would be in $F$; hence say $X-C \notin F$, then $X-C \subset W$, but then $K=X-W \subset C$, and so $K=C$. 
It is well known that the irreducible subsets of a topological space $X$ correspond to the points in the category of set-valued sheaves on $X$ ([1], 7.24). We now have points corresponding to the constrained filters on $O X$. This is a gain, because irreducible subsets live only in a topological space, whereas constrained filters can be defined on any lattice.

We will denote a site $([1], 0.32)$ by $(C, T)$ where $\mathcal{C}$ is the small category and $T$ is a Grothendieck topology on $\mathcal{C}$. The set valued sheaves on $(C, T)$ will be denoted by $\operatorname{Shv}(C, T)$.

THEOREM 1.6. Let $L$ be a strongly distributive lattice and let $T$ be the canonical topology on $L$. Then the points of $\operatorname{Shv}(C, T)$ are in bijection with the constrained filters on $L$.

Proof. Constrained filters to points. The following diagram will be useful for reference in what follows:

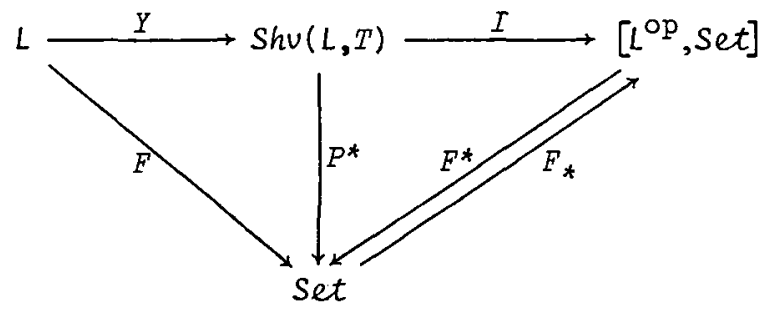

Given a filter on $L$ we take its characteristic functor $F: L \rightarrow$ Set (as in Theorem 1.1). This is left exact and hence has a left exact left Kan extension $F^{*}$ with a right adjoint $F_{*}$. Let $P^{*}=F^{*} I$. Then $P^{*}$ is left exact and will be the inverse image of a point if $F_{*}$ lands in $\operatorname{Shv}(L, T)$. The density of $Y$ says that if a functor $P: \operatorname{Shv}(L, T) \rightarrow \operatorname{Set}$ preserves colimits then it is of the form $E^{*} I$. Now suppose $F$ is constrained. We must show $E_{*}$ lands in $\operatorname{Shv}(L, T)$. That is we must show that the functor $F_{*} A=\operatorname{Set}(F-, A)$ is a sheaf for all sets $A$. Let $U$ be an object of $L$ and let $\left\{U_{\alpha}\right\}$ be a covering of $U$. Let $U \& F$ for all $\alpha$. Hence there is only one element of $\operatorname{Set}(F U, A)=1$ and one of $\operatorname{Set}\left(F U_{\alpha}, A\right)$, and so the sheaf property is trivial. Suppose $U \in F$. Since $F$ is constrained, at least one $U_{\alpha} \in F$. Given $s_{\alpha} \in \operatorname{Set}\left(F U_{\alpha}, A\right)$ for each $\alpha$ with the usual consistency, each $s_{\alpha}: 1 \rightarrow A$ corresponds to 
the same point in $A$, say $a \in A$, for all $U_{\alpha} \in F$. Hence let $s: F U=1 \rightarrow A$ correspond to the point $a$ and $E_{*} A$ is a sheaf.

Points to filters. Suppose $P_{*}: \operatorname{Set} \rightarrow \operatorname{Shv}(L, T)$ is a point with left exact left adjoint $P^{*}$. Let $F=P^{*} Y: L \rightarrow$ Set. Then $F$ is a left exact functor. Then $F=\{U \in L \mid F U=1\}$ is a filter on $L$. This filter is constrained because $\left\{U_{\alpha}\right\}$ is a family of objects not in $F$; then $F U_{\alpha}=0$ for all $\alpha$. But

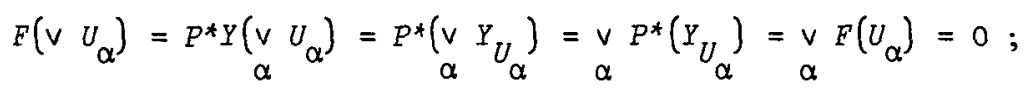

hence $v_{\alpha} U_{\alpha} \notin F$. Here we denote by $y_{U}$ the image of $U$ under $Y$. The remark that $P^{*}$ was of the form $F^{*} I$ gives that the two processes are inverses of each other.

A point where this is an overlap between Theorems 1.1 and 1.6 is the case when $L$ is a complete Heyting algebra, for then $L$ is the poset of subobjects of $1[1,5.37]$.

COROLLARY 1.7. Let $L$ be a complete Heyting algebra. Then the points of $\operatorname{Shv}(L, T)$ are the constrained filters on the poset of subobjects of 1 (where $T$ is the canonical topology).

Proof. This is a summary of the remark above and Theorem 1.6 .

In general the two conditions on the topology needed to force constrained filters to be points are:

1. representables are sheaves;

2. the Yoneda functor $Y: L \rightarrow \operatorname{Shv}(L, T)$ preserves unions. For example a corollary to the proof of Theorem 1.6 is:

COROLLARY 1.8. Let $L$ be a distributive lattice and let $T$ be the topology whose coverings of $U$ are finite families $\left\{U_{i}\right\}_{1}^{n}$. Then the points of $\operatorname{Shv}(L, T)$ are the constrained filters on $L$.

The result of Corollary 1.8 is part of the "folk-lore" of topos theory and can be proven directly. This does not seem to appear in the literature. 


\section{References}

[1] P.T. Johnstone, Topos theory (London Mathematical Society Monographs, 10. Academic Press, London, New York, San Francisco, 1977).

[2] Saunders Mac Lane, Categories for the working mathematicion (Graduate Texts in Mathematics, 5. Springer-Verlag, New York, Heidelberg, Berlin, 1971).

[3] Friedrich UImer, "Locally $\alpha$-presentable and locally $\alpha$-generated categories", Reports of the Miduest Category Seminar V, 230-247 (Lecture Notes in Mathematics, 195. Springer-Verlag, Berlin, Heidelberg, New York, 1971).

School of Mathematics and Physics, Macquarie University, North Ryde, New South Wales. 